\title{
SNV reoccurrence in multiple regions in the genome of 2019-nCoV
}

\author{
Xinjun $\mathrm{He}^{1, *}$, Ph.D., Wei Yue ${ }^{1}$, M.S., Jun Yan ${ }^{1}$, Ph.D.
}

\section{Affiliation:}

Nanjing YiGenCloud Institute

211 Pubin Rd. Nanjing Jiangbei New Area, Nanjing, China, 210000

* Correspondence: xinjun.he@yigencloud.cn

\begin{abstract}
:
The rapid development of 2019-2020 Wuhan seafood market pneumonia currently posed a major public health concern in China. Genome sequencing identified a novel beta-coronavirus closely related to SARS-CoV, named 2019-nCoV by WHO, as the cause of this pandemic disease. Viruses with single stranded RNA genome are prone to evolve quickly by accumulation of mutations, such as SNV, INDEL and cross viral recombination, aiding fast transmission among hosts and cross species. Here we collected related genome sequences and investigated variations shared by different strains of 2019-nCoV, identified reoccurrence of SNV mutations in clusters of patients, an indication of rapid evolution of $2019-\mathrm{nCoV}$ at the transmission from animal host to human. The information collected herein would help to understand the dynamics of current pandemic.
\end{abstract}

\section{Key words:}

2019-nCoV, SARS-CoV-2, novel corona virus, Wuhan pneumonia 


\section{Introduction:}

As of Feb. $2^{\text {nd }}, 2020$, the 2019-2020 Wuhan seafood market pneumonia pandemic has reached more than 17,000 infected cases and caused 361 deaths in China (information obtained from the website publication of the National Health Commission of China). It currently posed a major public health concern both in China and internationally with rapid development (1-7).

A quick search of the cause resulted in the identification of a novel coronavirus, named 2019$\mathrm{nCoV}$, which has not been previously reported, in the subgenus of sarbecovirus. DNA sequencing indicated that this coronavirus contained $\sim 30 \mathrm{~kb}$ of single strand RNA genome (ssRNA) (GenBank: MN908947, posting on Virological.org website, communicated by Edward C. Holmes, University of Sydney on behalf of the consortium led by Professor YongZhen Zhang, Fudan University, Shanghai, http://virological.org/t/novel-2019-coronavirusgenome/319)(8). Multiple studies analyzed different strains of 2019-nCoV revealed its close relationship with SARS-CoV that caused 2002-2003 SARS pandemic pneumonia, and with several bat SARS-CoV related genomes, supporting the argument that 2019-nCoV may have transmitted from bat to human as well $(2,6,9)$. In-depth phylogenic analysis indicated that 2019-nCoV shares $96 \%$ identity with a bat coronavirus strain RaTG13 and 79\% of SARS-CoV $(6,10)$. It has been evidenced that frequent recombination happens among related coronavirus and fast evolution of 2019-nCoV genome with mutations has been proposed after among human transmission (10-14). Here we investigated publicly available 2019-nCoV genome sequences for such evidence.

\section{Results:}

We downloaded 53 genome sequences from 2019-nCoV and SARS, SARS-like virus sequences from GenBank, National Genomics Data Center of Beijing Institute of Genomics 
(BIGD), and Global Initiative on Sharing All Influenza Data (GISAID) databases (Table 1). 53 complete genome sequences were then aligned with ClustalO algorithms online server at EBI (https://www.ebi.ac.uk/Tools/msa/clustalo/)(15). Alignments of sequences then were imported into JalViewer 2.0.11.0(16) for visualization. We also downloaded the metadata and mutation mapping data against 2019-nCoV reference genome (GenBank: NC_045512) from 2019 Novel Coronavirus Resource of BIGD to gain information of genome variations.

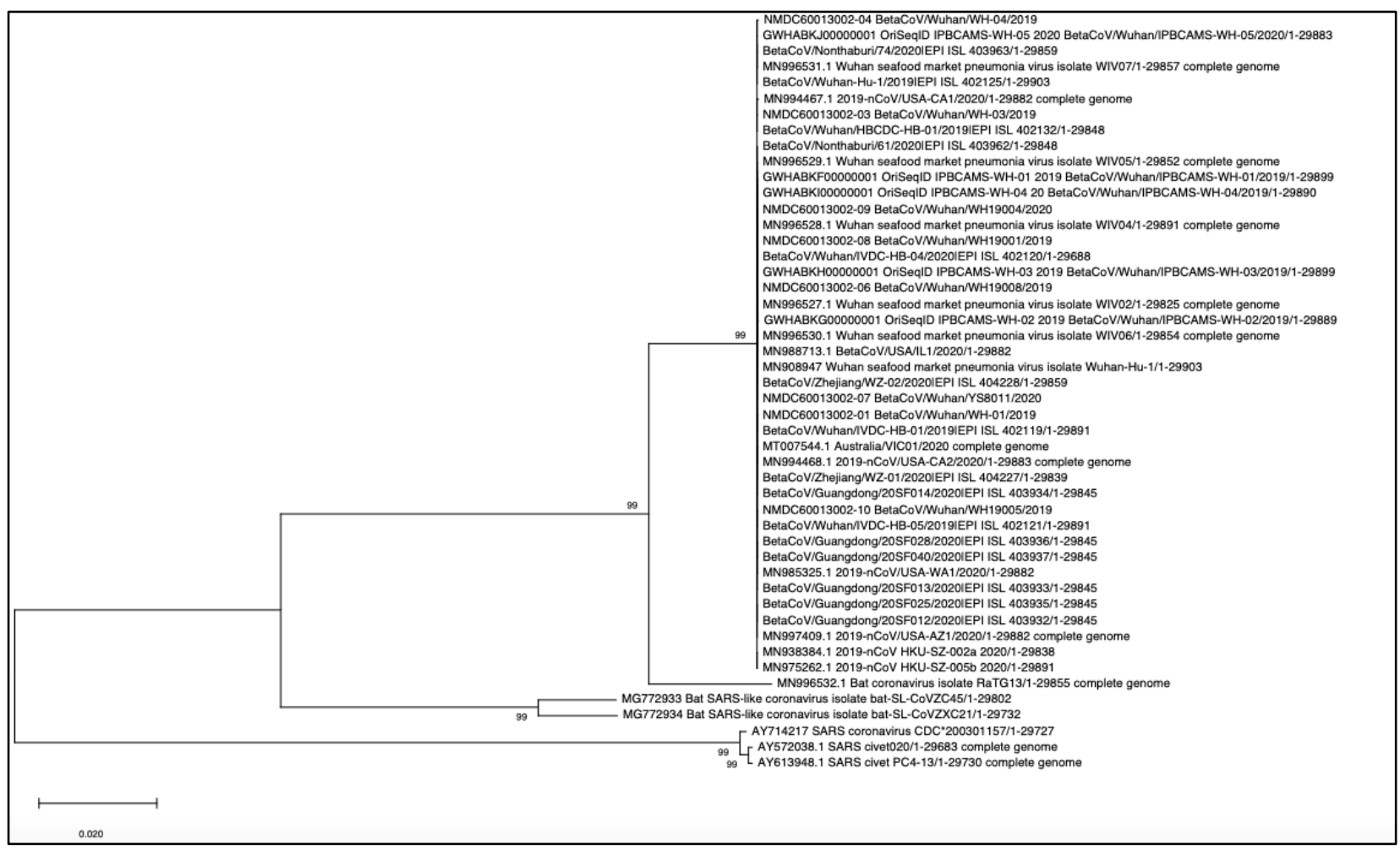

Figure 1. Phylogenetic tree reconstruction of 2019-nCoV with related SARS viral genomes. Whole genome sequences of 2019-nCoV, two bat SARS-like sequence, RaTG13, two civet SARS and one SARS-CoV from human were aligned and phylogenetic tree was reconstructed with NJ with 100 bootstrap replications. SARS sequences were used to root the tree. 
Table 1. 2019-nCoV Genome Sequences (Obtained from BIGD 2020-02-02 for genome wide mutation analysis)*

\begin{tabular}{|c|c|c|c|c|c|c|}
\hline Virus Strain Name & Accession ID & Data Source & Related ID & Sample Colle & ielocation & Submitting Lab \\
\hline $2019-n C o V W H U 011$ & MN988668 & & EPI_ISL_406716 & & & \\
\hline 2019-nCoVWHUO2 & MN988B669 & Gengank & EPI_ISL_406717 & $2020-01-02$ & China/Hubei/Wuhan & State Key Laboratory of Virology, Wuhan University \\
\hline 2019-nCoV_HKU-SZ-002a_2020 & MN938384 & Gengank & EPI_ISL_406030 & $2020-1$ & China / Guangdong / Shenzher & University of Hong Kong-Shenzhen Hospital \\
\hline 2019-nCoV_HKU-SZ-005b_2020 & MN975262 & Gengank & EPI_ISL_-405839 & $2020-1$ & China / Guangdong / Shenzhen & University of Hong Kong-Shenzhen Hospital \\
\hline 2019-nCoV/USA-AZ1/2020 & MN997409 & Gengank & EPI_ISL_-406223 & 2020-01-22 & USA/Arizona/Phoenix & Division of Viral Diseases, Centers for Disease Control and Prevention \\
\hline 2019-nCoV/USA-CA1/2020 & MN994467 & GenBank & EPI_ISL_406034 & 2020-01-23 & USA/California/Los Angeles & Division of Viral Diseases, Centers for Disease Control and Prevention \\
\hline 2019-nCoV/USACAZ/2020 & MN994468 & GenBank & EPI_ISL_406036 & 2020-01-22 & USA/California/Orange County & Division of Viral Diseases, Centers for Disease Control and Prevention \\
\hline 2019-nCoV/USA-IL1/2020 & MN988713 & GenBank & EPI_ISL_404253 & 2020-01-21 & USA/llinois/Chicago & IL Department of Public Health Chicago Laboratory \\
\hline $\begin{array}{l}\text { 2019-nCoV/USA-WA1/2020 } \\
\text { BetaCoV/Foshan/20SF207/2020 }\end{array}$ & $\begin{array}{l}\text { MN9855325 } \\
\text { EPI 15L 406534 }\end{array}$ & $\begin{array}{l}\text { Gennank } \\
\text { GISAD }\end{array}$ & EPI_ISL_404895 & $\begin{array}{l}2020-01-19 \\
20201-22\end{array}$ & $\begin{array}{l}\text { USA/Washington / Snohomish County } \\
\text { China / Gavengdong Province }\end{array}$ & 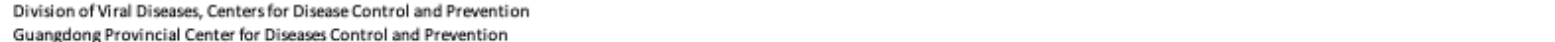 \\
\hline BetaCoV/Foshan/20SF210/2020 & EPIISL_-406535 & GISAID & & $2020-01-22$ & $\begin{array}{l}\text { China/ Guangdong Province } \\
\text { Conce }\end{array}$ & Guangdong Provincial Center for Diseases Control and Prevention \\
\hline BetacoV/Foshan/20SF211/2020 & EPI_ISL_406536 & GISAID & & $2020-01-22$ & China/ Guangdong Province & Guangdong Provincial Center for Diseases Control and Prevention \\
\hline BetacoV/Guangdong/20SF012/2020 & EPI_ISL_-403932 & GISAID & & $2020-01-14$ & China/Guangdong Province/Shenzhen Ci & Ci Department of Microbiology, Guangdong Provincial Center for Diseases Contorl and Prevention \\
\hline BetaCoV/Guangdong/205FO13/2020 & EP__ISL_-403933 & GISAID & & $2020-01-15$ & China/Guangdong Province/ Shenzhen Ci & ci Department of Microbiology, Guangdong Provincial Center for Diseases Contorl and Prevention \\
\hline BetaCoV/Guangdong/205F014/2020 & EPI_ISL_403934 & GISAID & & 2020-01-15 & China/Guangdong Province/Shenzhen Ci & Ci Department of Microbiology, Guangdong Provincial Center for Diseases Contorl and Prevention \\
\hline BetaCoV/Guangdong/205F025/2020 & EPI_ISL_403935 & GISAID & & 2020-01-15 & China/Guangdong Province/ Shenthen Ci & ci Department of Microbiology, Guangdong Provincial Center for Diseases Contorl and Prevention \\
\hline BetacoV/Guangdong/205Fo28/2020 & EPI_ISL_-_403936 & GISAID & & 2020-01-17 & China/ Guangdong Province/ Zhuhai City & y Department of Microbiology, Guangdong Provincial Center for Diseases Contorl Ind Prevention \\
\hline 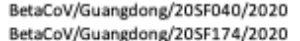 & $\begin{array}{l}\text { EPI_ISL_403937 } \\
\text { EPIS__06531 }\end{array}$ & GISAD & & 2020-01-18 $2020-182$ & $\begin{array}{l}\text { China / Guangdong Province/Zhuhai City } \\
\text { China/ Gavedong Province. }\end{array}$ & 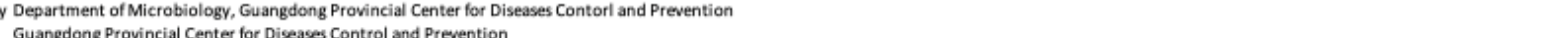 \\
\hline 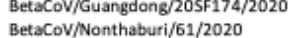 & 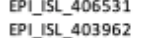 & GISAID & & $2020-1 \cdot-82$ & $\begin{array}{l}\text { China/Guangdong grovince } \\
\text { Thailand/Nonthaburi Province }\end{array}$ & $\begin{array}{l}\text { Guangdong Provincial Centef for Diseases control and Prevention } \\
\text { Department of Medical Sciences, Ministry of Public Health, Thailand; Thai Red Cross Emerging Intec }\end{array}$ \\
\hline BetaCoV/Nonthaburi/74/2020 & EPI_ISL_-403963 & GISAID & & 2020-1-13 & Thailand/Nonthaburi Province & 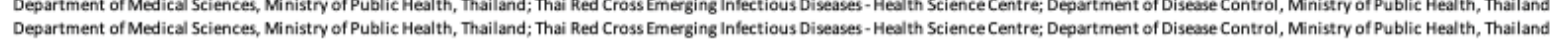 \\
\hline BetaCoV/Shenzhen/52TH-001/2020 & EPI_ISL_406592 & GISAID & & $2020-01-13$ & China/Guangdong Province/Shenzhen Ci & is Shenzhen Key Laboratory of Pathogen and Immunity, National Clinical Research Center for Infectious Disease, Shenthen Third People's Hospital \\
\hline Betacov/Shenzhen/5zTH-002/2020 & EPI_ISL_406593 & GISAID & & $2020-01-13$ & China/ Guangdong Province/ Shenzhen Ci & I I Shenzhen Key Laboratory of Pathogen and Immunity, National Clinical Research Center for Infectious Disease, Shenzhen Third People's Hospital \\
\hline BetacoV/Shenzhen//52TH-003/2020 & EPI_ISL_406594 & GISAID & & 2020-01-16 & China/Guangdong Province/Shenzhen Ci & is Shenzhen Key Laboratory of Pathogen and Immunity, National Clinical Research Center for Infectious Disease, Shennthen Third People's Hospital \\
\hline BetacoV/Shenzhen//52TH-004/2020 & EPI_ISL_406595 & GISAID & & 2020-01-16 & China/Guangdong Province/ Shenzhen Ci & is Shenzhen Key Laboratory of Pathogen and Immunity, National Clinical Research Center for Infectious Disease, Shenzhen Third People's's Hospital \\
\hline BetacoV/Wuhan/HBCDC-HB-01/2019 & EPI_ISL_-402132 & GISAID & & 2019-12-30 & China/Hubei Province & Hubei Provincial Center for Disesse Control and Prevention \\
\hline BetaCoV/Wuhan//PBCAMS-WH-01/20 & GWHABKFO00000 & Genome Wareh & E EPI_ISL_402123 & 2019-12-23 & China/Hubei Province/Wuhan City & Institute of Pathogen Biology, Chinese Academy of Medical Sciences \& Peking Union Medical College; Vision Medicals Co., Ltd \\
\hline BetaCoV/Nuhan/PBCAMS-WH-02/20 & GWHABKGO0000C & Genome Wareh & EPI_ISL_403931 & 2019-12-30 & China/Hubei Province/Wuhan City & Institute of Pathogen Biology, chinese Academy of Medical Sciences \& Peking Union Medical College \\
\hline BetaCoV/Nuhan//PBCAMS-WH-03/20 & & Genome Ware: & -403930 & 2019-12-30 & China/Hubei Province/Wuhan City & Institute of Pathogen Biology, Chinese Academy of Medical Sciences \& Peking \\
\hline & & & & 2019-12-30 & China / Hubei Province/Wuhan City & Institute of Pathogen Biology, Chinese Academy of Medical Sciences \& Peking Union Medical College \\
\hline BetacoV/Wuhan/PBCAMS-WH-05/20 & GWHABKJ $0000000 \mathrm{C}$ & & EPI_ISL_-_403928 & $2020-1-1$ & China/Hubei Province/Wuhan City & Institute of Pathogen Biologyr, Chinese Academy of Medical Sciences \& Peking Union Medical College; China National Center for Bioinformation \\
\hline BetacoV/Wuhan/VDC-18-01/2019 & EPI_ISL_402119 & GISAID & & 2019-12-30 & China/ Hubei Province/Wuhan city & National Institute for Viral Disease Control and Prevention, China CDC \\
\hline BetacoV/Nuhan/livoC+18-04/2020 & EPI_ISL_-402120 & GISAID & & $2020-1 \cdot 1$ & China/Hubei Province/ Wuhan City & National Institute for Viral Disease Control and Prevention, China CDC \\
\hline BetaCoV/Nuhan/ivDC-18-05/2019 & EPI_ISL_-402121 & GISAID & & 2019-12-30 & China/Hubei Province/Wuhan City & National Institute for Viral Disease Control and Prevention, China CDC \\
\hline BetacoV/Nuhan/WH-01/2019 & NMDC60013002-01 & INMDC & EPI_ISL_406798, CN CN & u 2019-12-26 & China/Hubei Province/Wuhan City & BGI PathoGenesis Pharmaceutical Technology Co., Ltd; China CDC; Shandong First Medical University \& Shandong Academy of Medical Sciences; Hub \\
\hline BetacoV/Wuhan/WH-03/2019 & NMDC60013002-03 & NMDC & EPL1ILL-406800, CN & $\begin{array}{ll}42020-01-01 \\
\mu 2020-015\end{array}$ & 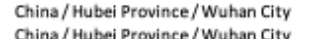 & 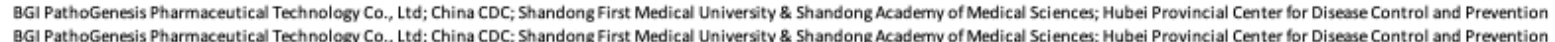 \\
\hline Betacov/Wuhan/WH-04/2019 & $\begin{array}{l}\text { NMDC60013002-04 } \\
\text { NMDC600130208 }\end{array}$ & INMDC & EPI_ISL_406801, CI & $\begin{array}{l}42020-0105 \\
2019-12-30\end{array}$ & $\begin{array}{l}\text { China/Hubei Province/Wuhan City } \\
\text { Chinas/Hubei Province/Wuban }\end{array}$ & 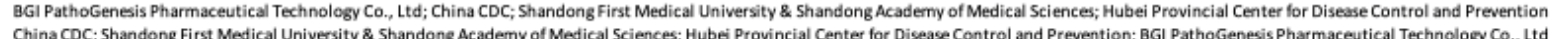 \\
\hline BetacoV/Nuhan/WH19001/2019 & 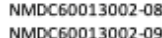 & NMDC & & 2019-12-30 2020 & $\begin{array}{l}\text { China//Hubei Province/Wuhan City } \\
\text { Chinas Hubei Province/Wutan }\end{array}$ & 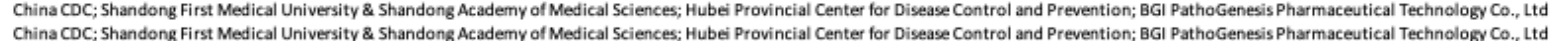 \\
\hline $\begin{array}{l}\text { BetecCov/Wunar/WH19004/2020 } \\
\text { Betacov/Whan/WH19005/2019 }\end{array}$ & $\begin{array}{l}\text { NMDD600130022-09 } \\
\text { NMDC60013002-10 }\end{array}$ & $\begin{array}{l}\text { NMDCC } \\
\text { NMDC }\end{array}$ & & 2019-12-30 & 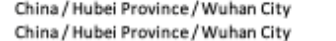 & 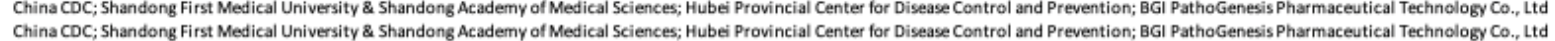 \\
\hline BetaCoV/Wuhan/WH19008/2019 & NMDC60013002-06 106 & NMDC & & 2019-12-30 & China/Hubei Province/Wuhan City & $\begin{array}{c}C h i n a \\
C D C\end{array}$ \\
\hline BetacoV/Wuhan/158011/2020 & NMDC60013002-07 & NMDC & & 2020-01-07 & China/Hubei Province/Wuhan City & China CDC; Shandong First Medical University \& Shandong Academy of Medical Sciences; Hubei Provincial Center for Disease Control and Prevention; BG PathoGenesis Pharmaceutical Technology Co., ttd \\
\hline TG13 & GWHABKPO000000 & & EEPI_ISL_402131, & & China/Yunnan/Pu'et & CAS Key Laboratory of Special Pathogens and Biosatety and Center for Emerging Infectious Diseases, Wuhan Institute of Virology, chinese Academy of Sciences \\
\hline wivo2 & GWHABKKO000000 & & EPIIIS & & China/Hubei / Wuhan & CAS Key Laboratory of Special Pathogens and Biosafety and Center for Emerging Infectious Diseases, wuhan Institute of Virology, Chinese Academy of Sciences \\
\hline wivo4 & GWHABKLO000000 & c Genome W & & & China / Hubei / Wuhan & CAS Key Laboratory of Special Pathogens and Biosatety and Center for Emerging Infectious Diseases, Wuhan Institute of Virology, Chinese Acadeny of Sciences \\
\hline Wivos & GWHABKMO000000 & Genome W: & EEPI_ISL_40 & & China / Hubei / Wuhan & CAS Key Laboratory of Special Pathogens and Biosafety and Center for Emerging Infectious Diseases, Wuhan Institute of Virology, Chinese Academy of Sciences \\
\hline WIVo6 & GWHABKNO000000 & NGenome Wa & EEPI_ISL_402 & & China/Hubei / Wuhan & CAS Key Laboratory of Special Pathogens and Biosafety and Center for Emerging Infectious Diseases, Wuhan Institute of Virology, Chinese Academy of Sciences \\
\hline $\begin{array}{l}\text { Wivo7 } \\
\text { Wuhan Hu-1 }\end{array}$ & $\begin{array}{l}\text { GWHABBO0000000 } \\
\text { MN908947 }\end{array}$ & 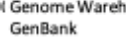 & $\begin{array}{l}\text { EPIIISL } 402130, \\
\text { NC } 045512\end{array}$ & $\begin{array}{l}N 2019-12-30 \\
2012\end{array}$ & $\begin{array}{l}\text { China/ Hubbei Wuhan /Wuhan City } \\
\text { China/Hubei Province/ Whan }\end{array}$ & 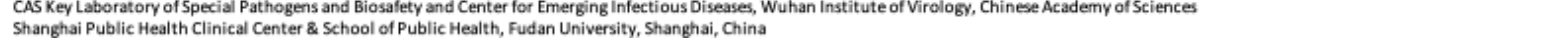 \\
\hline
\end{tabular}

*The following 4 sequences were obtained from GenBank for analysis:

AY572038.1_SARS_civet020,/1-29683, AY714217_SARS_coronavirus_CDC\#200301157,/1-29727, MG772933_Bat_SARS-

like_coronavirus_isolate_bat-SL-CoVZC45,/1-29802, MG772934_Bat_SARS-like_coronavirus_isolate_bat-SL-CoVZXC21,/1-29732 
Whole genome sequence alignment of 2019-nCoV together with human SARS, civet SARS, several bat SARS like CoV related virus supported previous conclusion that 2019-nCoV most closely clusters with a novel bat RaTG13 coronavirus (GenBank: MN996532)(6) and two bat SARS-like CoV strains (GenBank: MG772933 and MG772934, Figure 1)(2, 14). However, there is very limited diversity among these sequenced 2019-nCoV genomes, in line with the notion that 2019-nCoV was a recent jump from its original host to human population. In addition, as there have been evidences that recombination frequently occurs within the family of betacoronavirus, rendering the accuracy of whole genome phylogeny analysis, we further analyzed the essential ORF1ab and Spike/S proteins. Results support the close relationship between bat RaTG13 strain and 2019-nCoV (Figure 2).

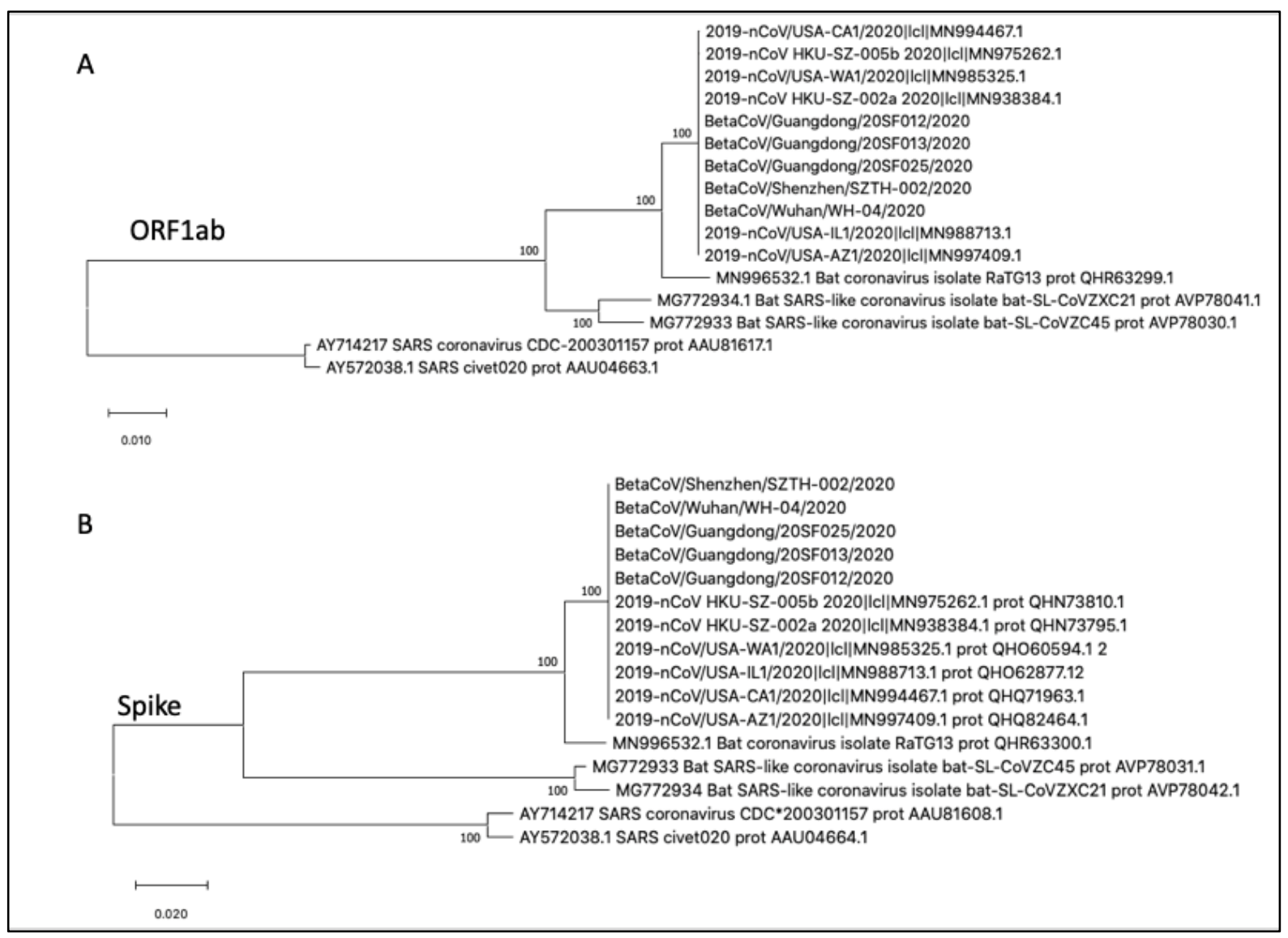

Figure 2. Phylogenetic trees reconstruction of ORF1ab and Spike/S protein. Protein sequence of eleven 2019-nCoV, RaTG13, two bat SARS-like, one civet and one human SARS viral 
proteins for ORF1ab (A) and Spike protein (B) were aligned and phylogenetic tree was reconstructed with $\mathrm{NJ}$ with 100 bootstrap replications. SARS sequences were used to root the tree.

After careful removal of false positive mutations obtained as of 2/19/2020 from BIGD, we collected shared SNVs of each sample against the reference genome (GenBank: NC_045512). We observed that there are at least $9 \mathrm{SNV}$, with at least two occurrences in these samples analyzed (Table 2).

Table 2. SNV identified with two or more occurrences

\begin{tabular}{ccccccc}
\hline SNV & Position & Ref & Alt & Mutation_type & AA_Change & Gene_Position \\
\hline SNV-8782 & 8782 & C & T & synonymous & QHD43415.1:p.2839S & ORF1ab:g.8517agCagT \\
SNV-28144 & 28144 & T & C & missense & QHD43422.1:p.84L>S & ORF8:g.251tTa>tCa \\
SNV-29095 & 29095 & C & T & synonymous & QHD43423.2:p.274F & N:g.822ttCttT \\
SNV-21707 & 21707 & C & T & missense & QHD43416.1:p.49H>Y & S:g.145Cat>Tat \\
SNV-24325 & 24325 & A & G & synonymous & QHD43416.1:p.921K & S:g.2763aaA>aaG \\
SNV-23569 & 23569 & T & C & synonymous & QHD43416.1:p.669G & S:g.2007ggT>ggC \\
SNV-28854 & 28854 & C & T & missense & QHD43423.2:p.194S>L & N:g.581tCa>tTa \\
SNV-20670 & 20670 & G & A & synonymous & QHD43415.1:p.6802A & ORF1ab:g.20406gcG>gcA \\
SNV-20679 & 20679 & G & A & synonymous & QHD43415.1:p.6805P & ORF1ab:g.20415ccG>ccA \\
\hline
\end{tabular}

These data were visually verified in sequence alignment (Figure 3). We noticed that SNV-

8782, SNV-28144 and SNV-29085 were shared by these samples:

BetaCoV/Guangdong/20SF012/2020|EPI_ISL_403932,

BetaCoV/Guangdong/20SF013/2020|EPI_ISL_403933,

BetaCoV/Guangdong/20SF025/2020|EPI_ISL_403935, MN938384.1_2019-nCoV_HKU-

SZ-002a_2020, MN975262.1_2019-nCoV_HKU-SZ-005b_2020, BetaCoV/Shenzhen/SZTH002/2020|EPI_ISL_406593, MN997409.1_2019-nCoV/USA-AZ1/2020, BetaCoV/Japan/TY- 
WK-012/2020|EPI_ISL_408665, BetaCoV/Japan/TY-WK-501/2020|EPI_ISL_408666 and BetaCoV/Japan/TY-WK-521/2020|EPI_ISL_408667.

SNV-8782 and SNV-28144 were shared additionally by samples: NMDC60013002-

04_BetaCoV/Wuhan/WH-04/2019, MN985325.1_2019-nCoV/USA-WA1/2020, MN994467.1_2019-nCoV/USA-CA1/2020 (Figure 3), and by additional strains obtained between 2/2/2020 and 2/19/2020 in BIGD database:

BetaCoV/Australia/NSW01/2020|EPI_ISL_407893, BetaCoV/Australia/QLD01/2020|EPI_ISL_407894, BetaCoV/Australia/QLD02/2020|EPI_ISL_407896, BetaCoV/Belgium/GHB03021/2020|EPI_ISL_407976, BetaCoV/Chongqing/YC01/2020|EPI_ISL_408478, BetaCoV/England/01/2020|EPI_ISL_407071, BetaCoV/England/02/2020|EPI_ISL_407073 BetaCoV/Japan/TY-WK-012/2020|EPI_ISL_408665, BetaCoV/Japan/TY-WK501/2020|EPI_ISL_408666, BetaCoV/Japan/TY-WK-521/2020|EPI_ISL_408667, BetaCoV/Sichuan/IVDC-SC-001/2020|EPI_ISL_408484, BetaCoV/Taiwan/NTU01/2020|EPI_ISL_408489, BetaCoV/USA/IL2/2020|EPI_ISL_410045, BetaCoV/Vietnam/VR0338142/2020|EPI_ISL_408668, BetaCoV/Yunnan/IVDC-YN-003/2020|EPI_ISL_408480. 


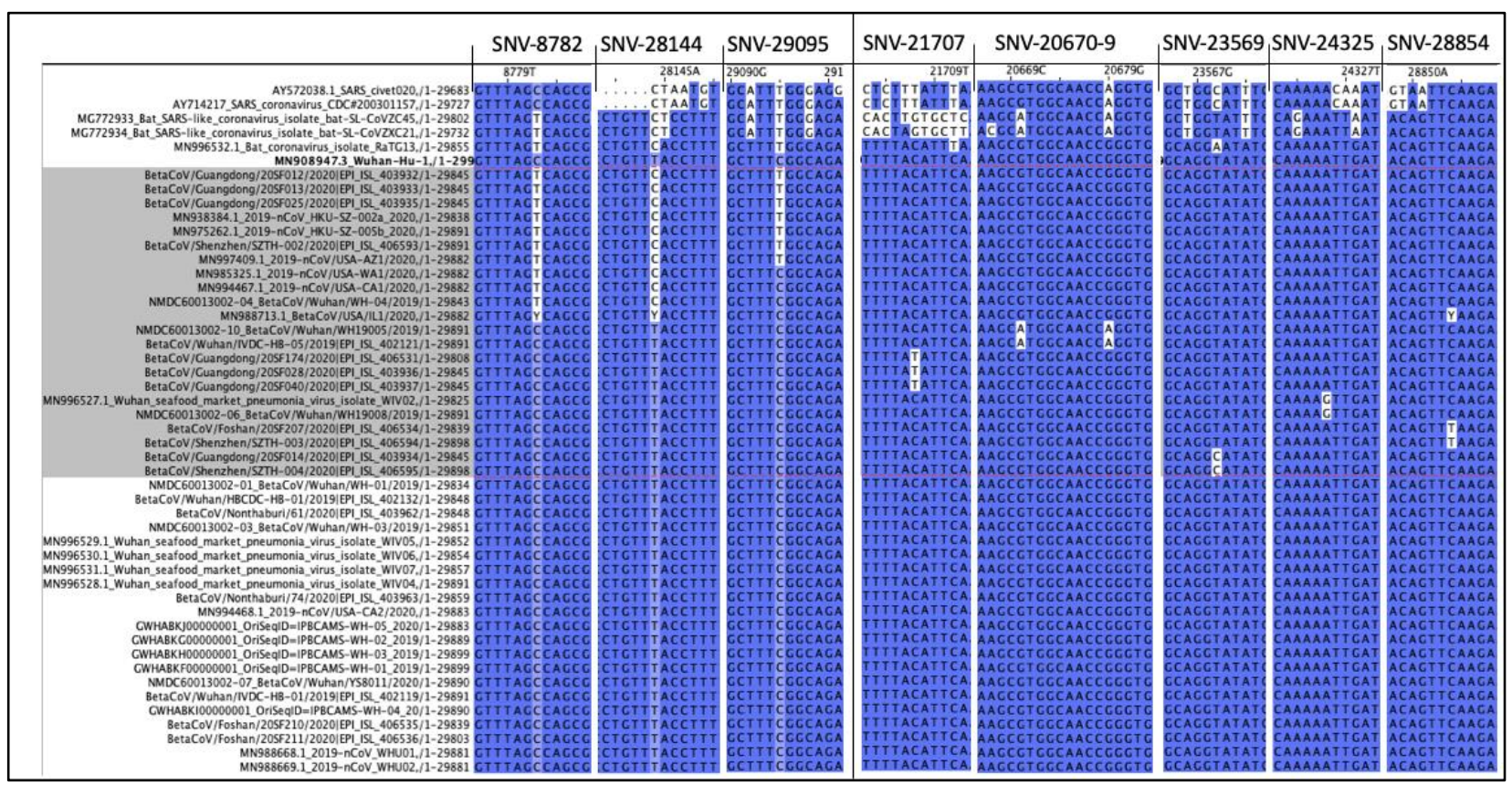

Figure 3. Sequence alignment of 2019-nCoV strains with SARS related viral genomes centered around SNV-8782, SNV-28144, SNV-29095, SNV-20670-9, SNV-21707, SNV24325 and SNV-28854. Sequences were aligned with strain names on the left. Each SNV were labeled on the top of alignment screenshot respectively. Consensus sequence were masked blue. Number denotes the location in reference genome.

Samples of MN938384.1_2019-nCoV_HKU-SZ-002a_2020, MN975262.1_2019nCoV_HKU-SZ-005b_2020, BetaCoV/Guangdong/20SF012/2020|EPI_ISL_403932, BetaCoV/Guangdong/20SF013/2020|EPI_ISL_403933,

BetaCoV/Guangdong/20SF025/2020|EPI_ISL_403935, BetaCoV/Shenzhen/SZTH002/2020|EPI_ISL_406593, were all collected from patients in Shenzhen, Guangdong province, indicating the infection was likely due to a local transmission in the area; Samples of NMDC60013002-04_BetaCoV/Wuhan/WH-04/2019, and internationally isolated MN985325.1_2019-nCoV/USA-WA1/2020 and MN994467.1_2019-nCoV/USA-CA1/2020 may also traced back to Wuhan travels, indicating SNV-8782 and SNV-28144 may be from parental viral ancestor and within which, SNV-29085 was derived. 
The above prediction was supported that MN938384.1_2019-nCoV_HKU-SZ-002a_2020 and MN975262.1_2019-nCoV_HKU-SZ-005b_2020 that have been reported as an example of cluster transmission (17), indicating either fast evolution of 2019-nCoV viral genome from a single common ancestor passed down into human population, or spillover of multiple common viral ancestors from the natural reservoir of virus host to human.

We also noticed that SNV-21707 are shared by three samples collected in Zhuhai, Guangdong province by the Guangdong Provincial Center for Diseases Control and Prevention between Jan. 17 and Jan. 22 of 2020: BetaCoV/Guangdong/20SF028/2020|EPI_ISL_403936, BetaCoV/Guangdong/20SF040/2020|EPI_ISL_403937, BetaCoV/Guangdong/20SF174/2020|EPI_ISL_406531, these are likely derived from a single cluster of infected patients. Similarly, SNV-20670-9, SNV-23569, SNV-28854 and SNV24325 were collected at the same location within a short time frame, such as SNV-24325 are shared by two samples: GWHABKK00000000-BetaCoV/Wuhan/WIV02/2019 and NMDC60013002-06_BetaCoV/Wuhan/WH19008/2019, which were collected on Dec. 30th of 2019 in Wuhan by two different labs, indicating that SNV may also have been derived from a common cluster of infected patients.

\section{Discussion:}

2019-nCoV contains a single positive single strand RNA genome of $~ 30 \mathrm{~kb}$ in size. 2019-nCoV has been shown to be closely related to SARS and SARS related virus in human and in natural bat reservoir at genomic sequences and amino acid sequences of coded protein levels from phylogeny analysis. 
It is expected to see that the ssRNA genome containing 2019-nCoV will evolve fast as it is transmitted among human population. Our data analysis showed, as of Feb. 2nd, we have observed multiple reoccurrence SNVs in individual positions of multiple strains along its genome. These sequence variations are unlikely to be sequencing errors and occur in multiple samples collected in different locations.

With publicly available information associated with the sequences in hand, we speculated that there may be at least two possibilities explaining this observation: (1) due to fast evolvement of viral genome in human from a single common virus ancestor, fast enough to obtain these SNVs to diverge from its common viral ancestor. (2) multiple virus ancestors were transmitted from its host to human at the time of cross species transmission. While we are unable to distinguish these two possibilities at this moment, but it will be clear with sequencing data accumulation and identification of its natural host for 2019-nCoV.

However, at this moment, the impact of these mutations among these viruses are not clear, close monitoring of the fast evolution of viral genome is needed that may aid patient care due to this pandemic coronavirus.

\section{Conflict of interest:}

The authors declare no conflicts of interest.

\section{Acknowledgements:}

The 2019-nCoV genomes were generously shared by scientists from but not limited to the following institutions: the Shanghai Public Health Clinical Center \& School of Public Health, Fudan University, Shanghai, China, at the National Institute for Viral Disease Control and 
Prevention, China CDC, Beijing, China, at the Institute of Pathogen Biology, Chinese Academy of Medical Sciences \& Peking Union Medical College, Beijing, China, at the Wuhan Institute of Virology, Chinese Academy of Sciences, Wuhan, China, at the Department of Microbiology, Zhejiang Provincial Center for Disease Control and Prevention, Hangzhou, China, at the Guangdong Provincial Center for Diseases Control and Prevention at the Department of Medical Sciences, at the Shenzhen Key Laboratory of Pathogen and Immunity, Shenzhen, China, at the National Institute of Health, Nonthaburi, Thailand, at the US Centers for Disease Control and Prevention, Atlanta, USA, at the Institut Pasteur, Paris, France, at the University of Melbourne, Peter Doherty Institute for Infection and Immunity, Melbourne, Australia, and at the Victorian Infectious Disease Reference Laboratory, Melbourne, Australia and additional institutions via GISAID. We gratefully acknowledge the Authors, Originating and Submitting laboratories of the genetic sequence and metadata made available through GenBank, BIGD and GISAID on which this research is based. We also thank Qian Tan and Lei Ye for their discussion on this manuscript preparation.

\section{References:}

1. Q. Li et al., Early Transmission Dynamics in Wuhan, China, of Novel CoronavirusInfected Pneumonia. N Engl J Med, (2020).

2. N. Zhu et al., A Novel Coronavirus from Patients with Pneumonia in China, 2019. N Engl J Med, (2020).

3. M. Bassetti, A. Vena, D. Roberto Giacobbe, The Novel Chinese Coronavirus (2019nCoV) Infections: challenges for fighting the storm. Eur J Clin Invest, e13209 (2020).

4. M. L. Holshue et al., First Case of 2019 Novel Coronavirus in the United States. $N$ Engl J Med, (2020).

5. C. Huang et al., Clinical features of patients infected with 2019 novel coronavirus in Wuhan, China. Lancet, (2020).

6. P. Zhou et al., Discovery of a novel coronavirus associated with the recent pneumonia outbreak in humans and its potential bat origin. bioRxiv, 2020.2001.2022.914952 (2020).

7. L. L. Ren et al., Identification of a novel coronavirus causing severe pneumonia in human: a descriptive study. Chin Med J (Engl), (2020).

8. F. Wu et al., A new coronavirus associated with human respiratory disease in China. Nature, (2020). 
9. C. Xiong, L. Jiang, Y. Chen, Q. Jiang, Evolution and variation of 2019-novel coronavirus. bioRxiv, 2020.2001.2030.926477 (2020).

10. J. F. Chan et al., Genomic characterization of the 2019 novel human-pathogenic coronavirus isolated from a patient with atypical pneumonia after visiting Wuhan. Emerg Microbes Infect 9, 221-236 (2020).

11. W. Ji, W. Wang, X. Zhao, J. Zai, X. Li, Homologous recombination within the spike glycoprotein of the newly identified coronavirus may boost cross-species transmission from snake to human. J Med Virol, (2020).

12. J. Cui, F. Li, Z. L. Shi, Origin and evolution of pathogenic coronaviruses. Nat Rev Microbiol 17, 181-192 (2019).

13. Y. Wang et al., Origin and Possible Genetic Recombination of the Middle East Respiratory Syndrome Coronavirus from the First Imported Case in China: Phylogenetics and Coalescence Analysis. mBio 6, e01280-01215 (2015).

14. D. Hu et al., Genomic characterization and infectivity of a novel SARS-like coronavirus in Chinese bats. Emerg Microbes Infect 7, 154 (2018).

15. F. Sievers et al., Fast, scalable generation of high-quality protein multiple sequence alignments using Clustal Omega. Mol Syst Biol 7, 539 (2011).

16. A. M. Waterhouse, J. B. Procter, D. M. Martin, M. Clamp, G. J. Barton, Jalview Version 2--a multiple sequence alignment editor and analysis workbench. Bioinformatics 25, 1189-1191 (2009).

17. J. F. Chan et al., A familial cluster of pneumonia associated with the 2019 novel coronavirus indicating person-to-person transmission: a study of a family cluster. Lancet, (2020). 